\title{
HANKEL TRANSFORMS AND VARIATION DIMINISHING KERNELS
}

BY I. I. HIRSCHMAN, JR.

Communicated by Walter Rudin, October 8, 1959

If $\phi(x)$ is a continuous function on $(-\infty, \infty)$ then we denote by $V[\phi]$ the number of variations of sign of $\phi(x)$ on $(-\infty, \infty)$. A measurable function $G(x)$ on $(-\infty, \infty)$ such that

$$
G(x) \geqq 0, \quad \int_{-\infty}^{\infty} G(x) d x=1,
$$

and such that

$$
V[G * \phi] \leqq V[\phi]
$$

for every bounded continuous $\phi$ will be called a variation diminishing *-kernel. Here

$$
G * \phi \cdot(x)=\int_{-\infty}^{\infty} G(x-y) \phi(y) d y .
$$

I. J. Schoenberg has proved that if $G$ is a variation diminishing *kernel then

$$
\int_{-\infty}^{\infty} G(x) e^{-i x t} d x
$$

is of the form

$$
\left[e^{c t^{2}+i b t} \prod_{k}\left(1-\frac{i t}{a_{k}}\right) e^{i t / a_{k}}\right]^{-1}
$$

where the $a_{k}$ 's are real and $\sum_{k} a_{\mathrm{k}}^{-2}$ is finite, $b$ is real, and $c$ is real and non-negative. Conversely every function of the form (2) is the Fourier transform of a variation diminishing $*$-kernel. See [8] and [9].

In the present note we will sketch an analogous theory in which certain convolutions of functions on $(0 ; \infty)$, associated with Hankel transforms replace the convolution (1).

Let $\gamma$ be fixed, $0 \leqq \gamma$. We define

$$
\begin{aligned}
T(x) & =2^{\gamma-1 / 2} \Gamma(\gamma+1 / 2) x^{1 / 2-\gamma} J_{\gamma-1 / 2}(x), \\
\mu(x) & =x^{2 \gamma+1} / 2^{\gamma+1 / 2} \Gamma(\gamma+3 / 2) .
\end{aligned}
$$

Let $L$ be the set of measurable functions $f(x)$ on $(0, \infty)$ for which $\int_{0}^{\infty}|f(x)| d \mu(x)$ is finite. For $f \in L$ we set 


$$
f^{\wedge}(t)=\int_{0}^{\infty} T(x t) f(x) d \mu(x) .
$$

$f^{\wedge}(t)$ is the Hankel transform (of index $\gamma$ ) of $f(x)$. Let

$$
D(x, y, z)=\frac{2^{3 \gamma-5 / 2} \Gamma(\gamma+1 / 2)^{2}}{\Gamma(\gamma) \pi^{1 / 2}}(x y z)^{-2 \gamma+1} A(x, y, z)^{2 \gamma-2}
$$

where $A(x, y, z)$ is the area of a triangle whose sides are $x, y, z$ if there is such a triangle and otherwise is zero. If $f(x)$ and $g(x)$ are defined on $(0, \infty)$ then we formally set

$$
f \# g \cdot(x)=\int_{0}^{\infty} \int_{0}^{\infty} f(y) g(z) D(x, y, z) d \mu(y) d \mu(z) .
$$

It can be verified that if $f, g \in L$ then $f \# g \in L$ and $(f \# g)^{\wedge}=f^{\wedge} \cdot g^{\wedge}$; that is, the Hankel transform (3) behaves in regard to the convolution \# exactly as does the Fourier transform with regard to ordinary convolution $*$ on the real line. This convolution associated with the Hankel transform was discovered by Delsarte [3] and [4]. See also the papers of Bochner [1] and [2] and the author [6].

If $\psi(x)$ is a continuous function on $(0, \infty)$ let $V[\psi]$ denote the number of changes of sign of $\psi(x)$ on $(0, \infty)$. A measurable function $H(x)$ on $(0, \infty)$ such that

$$
H(x) \geqq 0, \quad \int_{0}^{\infty} H(x) d \mu(x)=1,
$$

and such that

$$
V[H \# \psi] \leqq V[\psi]
$$

for every continuous bounded function $\psi$ on $(0, \infty)$ will be called a variation diminishing \#-kernel. Our principal result is the following.

THEOREM. If $H(x)$ is a variation diminishing \#-kernel then $H^{\wedge}(t)$ is of the form

$$
\left[e^{c t^{2}} \prod_{k}\left(1+\frac{t^{2}}{a_{k}^{2}}\right)\right]^{-1}
$$

where the $a_{k}$ 's are real and $\sum_{k} a_{k}^{-2}$ is finite, and where $c$ is non-negative. Conversely every function of the form (4) is the Hankel transform of a variation diminishing \#-kernel. 
The demonstration of this result follows very closely the pattern established by Schoenberg. It is to be noted that for $\gamma=0$, this theorem is contained in Schoenberg's theorem as a special case.

Many important integral transforms can be reduced to the form $f=G * \phi$ where $G$ is a variation diminishing *-kernel. Such transforms have a very extensive theory which is the subject of a book by $D$. V. Widder and the author [7]. It is evident that a parallel development can be carried through for the transforms $g=H \# \psi$. See also in this connection the paper by Fox [5].

\section{REFERENCES}

1. S. Bochner, Positive zonal functions on spheres, Proc. Nat. Acad. Sci. U.S.A. vol. 40 (1954) pp. 1141-1147.

2. - Sturm-Liouville and heat equations whose eigenfunctions are ultraspherical polynomials or associated Bessel functions, Proceedings of the Conference on Differential Equations, held at the University of Maryland, 1955, pp. 23-48.

3. J. Delsarte, Sur une extension de la formule de Taylor, J. Math. Pures Appl. (9) vol. 17 (1936) pp. 213-231.

4. - Une extension nouvelle de la throrie de fonctions presque periodiques de Bohr, Acta. Math. vol. 69 (1938) pp. 259-317.

5. C. Fox, The inversion of convolution transforms by differential operators, Proc. Amer. Math. Soc. vol. 4 (1953) pp. 880-887.

6. I. I. Hirschman, Jr., Harmonic analysis and ultraspherical polynomials, Symposium on Harmonic Analysis and Related Integral Transforms held at Cornell University, 1956, vol. 1.

7. I. I. Hirschman, Jr. and D. V. Widder, The convolution transform, Princeton, 1955.

8. I. J. Schoenberg, On Polya frequency functions I, J. Analyse Math. vol. 1 (1951) pp. 331-374.

9. - On Polya frequency functions II, Acta Sci. Math. Szeged vol. 12 (1950) pp. 97-106.

WASHINGTON UNIVERSITY 